PROCEEDINGS OF THE

AMERICAN MATHEMATICAL SOCIETY

Volume 130, Number 11, Pages 3283-3285

S 0002-9939(02)06475-4

Article electronically published on May 29, 2002

\title{
ON ANTOSIK'S LEMMA AND THE ANTOSIK-MIKUSINSKI BASIC MATRIX THEOREM
}

\author{
QU WENBO AND WU JUNDE
}

(Communicated by Jonathan M. Borwein)

\begin{abstract}
That Antosik's Lemma is not a special case of the Antosik-Mikusinski Basic Matrix Theorem will be shown and, an equivalent form of the Antosik-Mikusinski Basic Matrix Theorem will also be presented in this paper.
\end{abstract}

In [1] and [2], Antosik proved two results which are called the Antosik-Mikusinski Basic Matrix Theorem and Antosik's Lemma, respectively. The theorem and lemma have been proven to be quite effective in treating various topics in Functional Analysis and Set Function Theory [1]-[6]. In [6, 2.2], Swartz thought that Antosik's Lemma is a special case of the Antosik-Mikusinski Basic Matrix Theorem. In [7, Li Ronglu presented the Uniform Convergent Principle. Now, we will show that Swartz's conclusion is incorrect and, the Uniform Convergent Principle is an equivalent form of the Antosik-Mikusinski Basic Matrix Theorem.

Lemma 1 (Antosik). Let $G$ be an abelian topological group and $x_{i j} \in G$ for $i, j \in$ $\mathbf{N}$. Suppose that each strictly increasing sequence $\left\{m_{i}\right\}$ in $\mathbf{N}$ has a subsequence $\left\{n_{i}\right\}$ such that

(i) $\lim _{i} x_{n_{i} n_{j}}=0$ for all $j \in \mathbf{N}$, and

(ii) $\lim _{i} \sum_{j=1}^{\infty} x_{n_{i} n_{j}}=0$.

Then $\lim x_{i i}=0$.

Antosik observed that assumption (i) can be dropped if $G$ is a locally convex space and posed the problem ([2]) of whether (i) can also be dropped in general. In 8], Weber solved this problem showing that assumption (i) is in fact superfluous.

A direct consequence of Lemma 1 is as follows:

Corollary 1. Let $G$ be an abelian topological group and $z_{i j} \in G$ for $i, j \in \mathbf{N}$. Suppose that

(I) $\lim _{i} z_{i j}=0$ for each $j \in \mathbf{N}$, and

(II) for each strictly increasing sequence of positive integers $\left\{m_{j}\right\}$ in $\mathbf{N}$ there is a subsequence $\left\{n_{j}\right\}$ such that $\lim _{i} \sum_{j=1}^{\infty} z_{i n_{j}}=0$.

Then $\lim _{i} z_{i i}=0$.

Received by the editors June 20, 2000 and, in revised form, June 11, 2001.

2000 Mathematics Subject Classification. Primary $40 \mathrm{C} 05$.

Key words and phrases. Antosik lemma, basic matrix theorem, uniform convergence principle. This work was supported by the NSF of China. 
Now, we show that the following Antosik-Mikusinski Basic Matrix Theorem can be obtained from Corollary 1.

Theorem 1 (Antosik-Mikusinski). Let $G$ be an abelian topological group, $x_{i j} \in G$ for $i, j \in \mathbf{N}$. Suppose

(III) $\lim _{i} x_{i j}=x_{j}$ exists for each $j$, and

(IV) for each strictly increasing sequence of positive integers $\left\{m_{j}\right\}$ there is a subsequence $\left\{n_{j}\right\}$ such that $\left\{\sum_{j} x_{i n_{j}}\right\}_{i=1}^{\infty}$ is a Cauchy sequence.

Then $\lim _{i} x_{i j}=x_{j}$ uniformly for $j \in \mathbf{N}$. In particular, $\lim _{i} x_{i i}=0$.

Proof. If the conclusion fails, then there exist a closed, symmetric neighbourhood $V_{0}$ of 0 in $G$ and strictly increasing sequences of positive integers $\left\{p_{k}\right\}$ and $\left\{q_{k}\right\}$ such that

$$
x_{p_{k} q_{k}}-x_{q_{k}} \notin V_{0}
$$

for all $k$. Pick a closed, symmetric neighbourhood $V_{1}$ of 0 such that $V_{1}+V_{1} \subseteq V_{0}$. Note that $x_{p_{i} q_{j}}-x_{q_{j}} \rightarrow 0$ as $i \rightarrow \infty$ for $j \in \mathbf{N}$. Therefore, there exists a subsequence $\left\{m_{i}\right\}$ of $\left\{p_{i}\right\}$ such that

$$
x_{m_{i} q_{i}}-x_{q_{i}} \in V_{1}
$$

for $i \in \mathbf{N}$. We have

$$
x_{p_{i} q_{i}}-x_{q_{i}}=\left(x_{p_{i} q_{i}}-x_{m_{i} q_{i}}\right)+\left(x_{m_{i} q_{i}}-x_{q_{i}}\right) .
$$

Consider the matrix $\left(x_{p_{i} q_{j}}-x_{m_{i} q_{j}}\right)$ and note that the matrix satisfies conditions of Corollary 1. Consequently,

$$
x_{p_{i} q_{i}}-x_{m_{i} q_{i}} \rightarrow 0
$$

as $i \rightarrow \infty$, and

$$
x_{p_{i} q_{i}}-x_{m_{i} q_{i}} \in V_{1}
$$

for sufficiently large $i$. Hence, by (3) and (2)

$$
x_{p_{i} q_{i}}-x_{q_{i}} \in V_{1}+V_{1} \subseteq V_{0}
$$

for sufficiently large $i$. Which contradicts (1) and we established the result.

Thus, we have Lemma $1 \Rightarrow$ Corollary $1 \Longleftrightarrow$ Theorem 1 . Now, we show that Lemma 1 is not a special case of Theorem 1.

Example 1. Consider the matrix $\left(x_{i j}\right)$ such that $x_{i j}=0$ if $i \neq j+1$ and $x_{i i-1}=1$. Then $\left(x_{i j}\right)$ satisfies the assumptions of Lemma 1 . If Lemma 1 was a special case of Theorem 1, then the columns should converge to 0 uniformly. But they do not converge to 0 uniformly.

In this way, we have corrected the incorrect statement in $([6,2.2])$.

Now, we use Theorem 1 to prove Theorem 2 below.

Theorem 2 (Uniform Convergent Principle). Let $G$ be an abelian topological group and let $\Omega$ be a sequentially compact topological space. Let $\left\{f_{i}\right\}$ be a sequence of sequentially continuous $G$-valued functions defined on $\Omega$. If each strictly increasing sequence $\left\{m_{j}\right\}$ in $\mathbf{N}$ has a subsequence $\left\{n_{j}\right\}$ such that for each $\omega \in \Omega$, the series $\sum_{j} f_{n_{j}}(\omega)$ converges and $\sum_{j} f_{n_{j}}: \Omega \rightarrow G$ is sequentially continuous, then $\lim _{j} f_{j}(\omega)=0$ uniformly with respect to $\omega \in \Omega$. 
Proof. We will show that $f_{j}(\omega) \longrightarrow 0$ uniformly for $\omega$ in $\Omega$ or, equivalently, for each sequence $\left\{\omega_{i}\right\}$ in $\Omega$,

$$
f_{i}\left(\omega_{i}\right) \longrightarrow 0
$$

as $i \longrightarrow \infty$. Let $\left\{\omega_{i}\right\}$ be a sequence of $\Omega$. Since $\Omega$ is sequentially compact, there exists a subsequence $\left\{\omega_{n_{i}}\right\}$ of $\left\{\omega_{i}\right\}$ and $\omega_{0}$ such that $\omega_{n_{i}} \longrightarrow \omega_{0}$. Consider the matrix $\left(f_{n_{j}}\left(\omega_{n_{i}}\right)-f_{n_{j}}\left(\omega_{0}\right)\right)$. Note that the matrix satisfies conditions of Theorem 1. Therefore,

$$
f_{n_{i}}\left(\omega_{n_{i}}\right)-f_{n_{i}}\left(\omega_{0}\right) \longrightarrow 0 .
$$

Since $f_{n_{i}}\left(\omega_{0}\right) \longrightarrow 0$, we get $f_{n_{i}}\left(\omega_{n_{i}}\right) \longrightarrow 0$. Hence, by assumption conditions, (4) holds. Thus, Theorem $1 \Longrightarrow$ Theorem 2 .

Theorem 3. Theorem 2 is an equivalent form of Theorem 1.

Proof. Since Corollary $1 \Longleftrightarrow$ Theorem $1 \Longrightarrow$ Theorem 2, we only need to show that Theorem $2 \Longrightarrow$ Corollary 1 .

Indeed, assume the conditions of Corollary 1 are satisfied. Let $\Omega=\left\{\frac{1}{i}, 0\right\}_{i=1}^{\infty}$, for $x, y \in \Omega$, put $d(x, y)=|x-y|$. Then $(\Omega, d)$ is a sequentially compact topological space. Let $f_{j}: \Omega \longrightarrow G$ satisfy that if $\omega=\frac{1}{i}, f_{j}(\omega)=z_{i j}$; if $\omega=0, f_{j}(\omega)=$ 0 . It is easily shown that each $f_{j}$ is continuous, and for each strictly increasing sequence $\left\{m_{j}\right\}$ in $\mathbf{N}$ has subsequence $\left\{n_{j}\right\}$ such that for $i \in \mathbf{N}$, the series $\sum_{j} z_{i n_{j}}$ is convergent and $\lim _{i} \sum_{j} z_{i n_{j}}=0$. Thus, for each $\omega \in \Omega$, the series $\sum_{j} f_{n_{j}}(\omega)$ is convergent and $\sum_{j} f_{n_{j}}: \Omega \longrightarrow G$ is continuous. It follows from Theorem 2 that $\lim _{j} f_{j}(\omega)=0$ uniformly with respect to $\omega \in \Omega$. In particular, $\lim _{j} f_{j}\left(\frac{1}{j}\right)=$ $\lim _{j} z_{j j}=0$. So Corollary 1 is proved.

\section{ACKNOWLEDGEMENT}

The authors would like to thank Professor Antosik and Professor Li Ronglu for their useful remarks and suggestions.

\section{REFERENCES}

1. P. Antosik, C. Swartz, Matrix Methods in Analysis, Lecture Notes in Mathematics, 1113, Springer-Verlag, Berlin, 1985. MR 87b:46079

2. P. Antosik, A lemma on matrices and its applications, Contemp. Math. 52(1986), 89-95. MR 87f: 46022

3. Wu Junde, Li Ronglu, An Orlicz-Pettis theorem with applications to $\mathcal{A}$-spaces, Studia Sci. Math. Hungary. 35(1999), 353-358. MR 2001g:46004

4. Wu Junde, Li Ronglu, Unconditional convergent series on locally convex spaces, Taiwanese J. Math. (4)2000, 253-259. MR 2001b:46009

5. Wu Junde, Li Ronglu, Hypocontinuity and uniform boundedness for bilinear maps, Studia Sci. Math. Hungary. (35)1999, 133-138. MR 2000a:46003

6. C. Swartz, Infinite Matrices and the Gliding Hump, World Sci. Publ., Singapore, 1996. MR 98b:46002

7. Li Ronglu, Cho Minhyung, A uniform convergent principle, J. Harbin Institute of Technology 24(1992), 107-108. MR 94a:46016

8. H. Weber, A diagonal theorem. Answer to a question of Antosik, Bull. Polish Acad. Sci. Math., 41(1993), 95-102. MR 98c:22001

Department of Mathematics, Harbin Institute of Technology, Harbin 150006, PeoPle's Republic of China

Department of Mathematics, Zhejiang University, Hangzhou 310027, People's RepubLIC OF CHINA

E-mail address: WJD@math.zju.edu.cn 